\title{
Single-Photon Ionization Mass Spectrometry Using a Vacuum Ultraviolet Femtosecond Laser
}

\author{
Supporting information
}

\section{Thang Dinh Phan, ${ }^{\dagger}$ Adan $\mathrm{Li}^{\dagger}{ }^{\dagger \ddagger}$ Hiroshi Nakamura, ${ }^{\dagger}$ Tomoko Imasaka, ${ }^{\S \star}$ and Totaro Imasaka ${ }^{\dagger \#}$}

${ }^{\dagger}$ Division of International Strategy, Center of Future Chemistry, Kyushu University, 744 Motooka, Nishiku, Fukuoka 819-0395, Japan

ॠCollege of Environmental and Chemical Engineering, Yanshan University, Qinhuangdao 066004, China

${ }^{\S}$ Department of Environmental Design, Graduate School of Design, Kyushu University, 4-9-1, Shiobaru, Minami-ku, Fukuoka 815-8540, Japan

"Hikari Giken, Co., 2-10-30, Sakurazaka, Chuou-ku, Fukuoka 810-0024, Japan

* To whom correspondence should be addressed. E-mail: imasaka@design.kyushu-u.ac.jp 
Supporting information provides emission spectrum of Raman sidebands (Fig. S1), the dependence of the hydrogen gas pressure on the signal intensity of the Raman sidebands (Fig. S2), spectrograph of Raman sidebands (Fig. S3), and absorption spectra calculated for pentachlorobenzene and APAHs (Fig. $4 S)$.

Figure S1. Emission spectrum of the Raman sidebands generated by two-color FWRM (800 and 1200 $\mathrm{nm})$. The probe beam $(200 \mathrm{~nm})$ was interrupted in the experiment. The hydrogen gas pressure was adjusted at $1.05 \mathrm{~atm}$. F1, fundamental beam $(800 \mathrm{~nm})$; AS1, first anti-Stokes $(600 \mathrm{~nm})$; AS2, second antiStokes (480 nm); AS3, third anti-Stokes (400 nm); AS4, fourth anti-Stokes (343 nm) Raman sidebands.

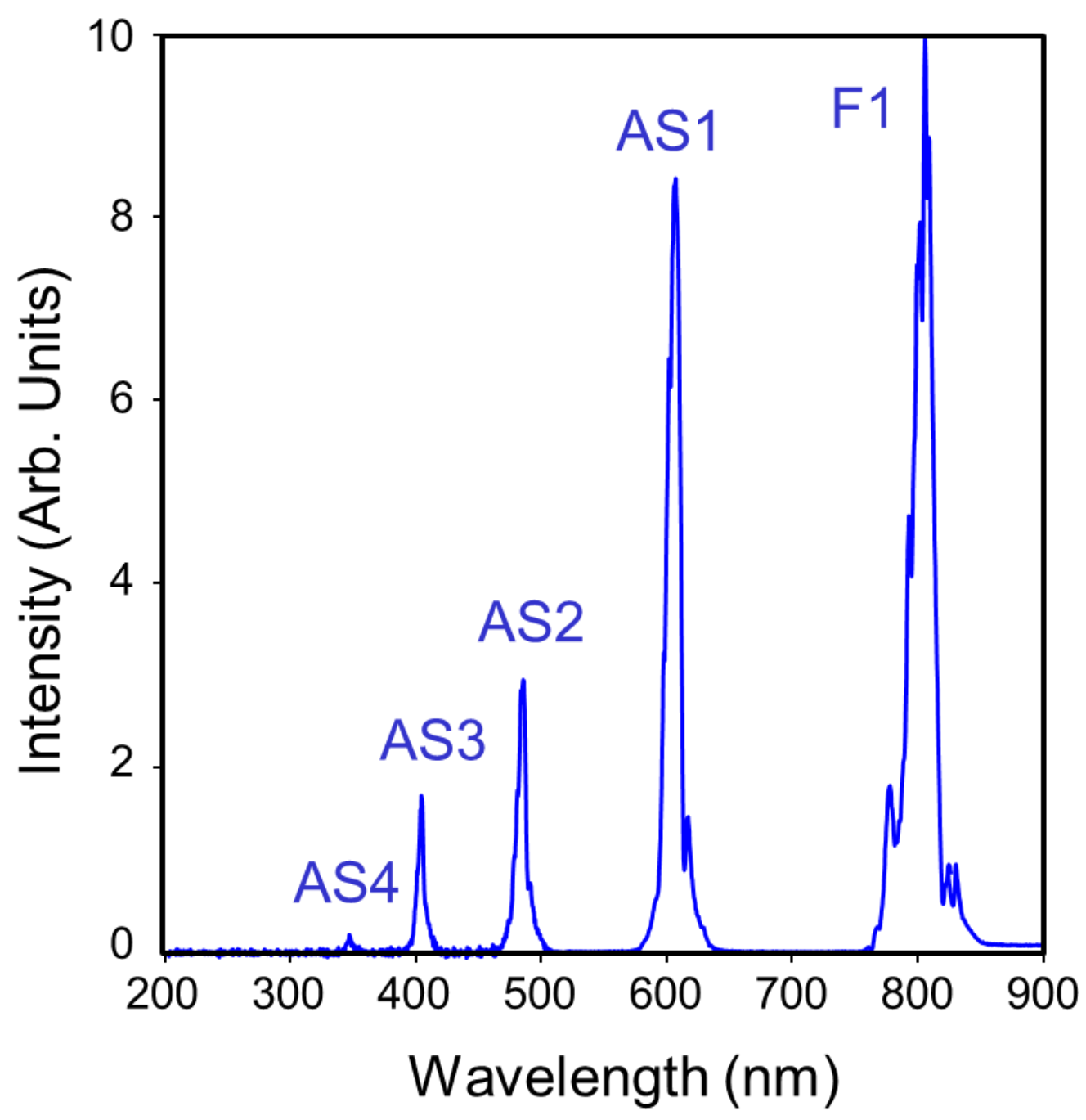


Figure S2. Dependence of hydrogen gas pressure on the intensity of the first anti-Stokes Raman sideband $(185 \mathrm{~nm})$. The intensity of the anti-Stokes sideband was optimal at $1.8 \mathrm{~atm}$, suggesting that phase matching in FWRM is required for generating the anti-Stokes emission.

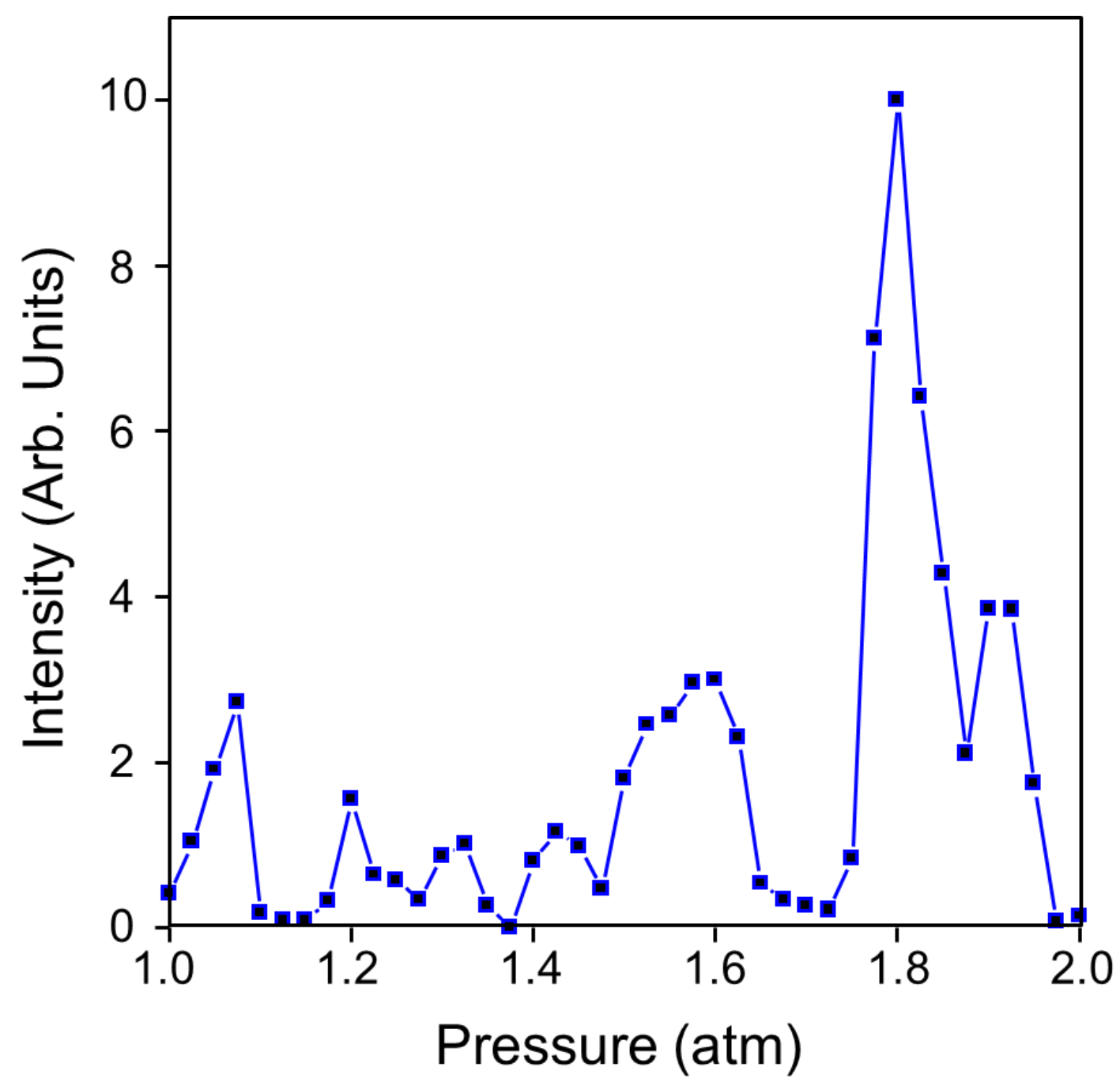


Figure S3. Spectrograph of the Raman sidebands measured at the exit window of the MS. An additional wedged substrate (a device shown by a broken line in Figure 2B) was inserted in the beam path after the exit window of the Raman cell to disperse the emissions at 185, 200, 218, and $240 \mathrm{~nm}$. The beam was focused with an aluminum concave mirror onto the analyte in the molecular beam (see Figure 2B). A phosphor material (sodium salicylate) was pasted on the inner surface of the exit window of MS to convert the UV-VUV emissions into the blue ones.

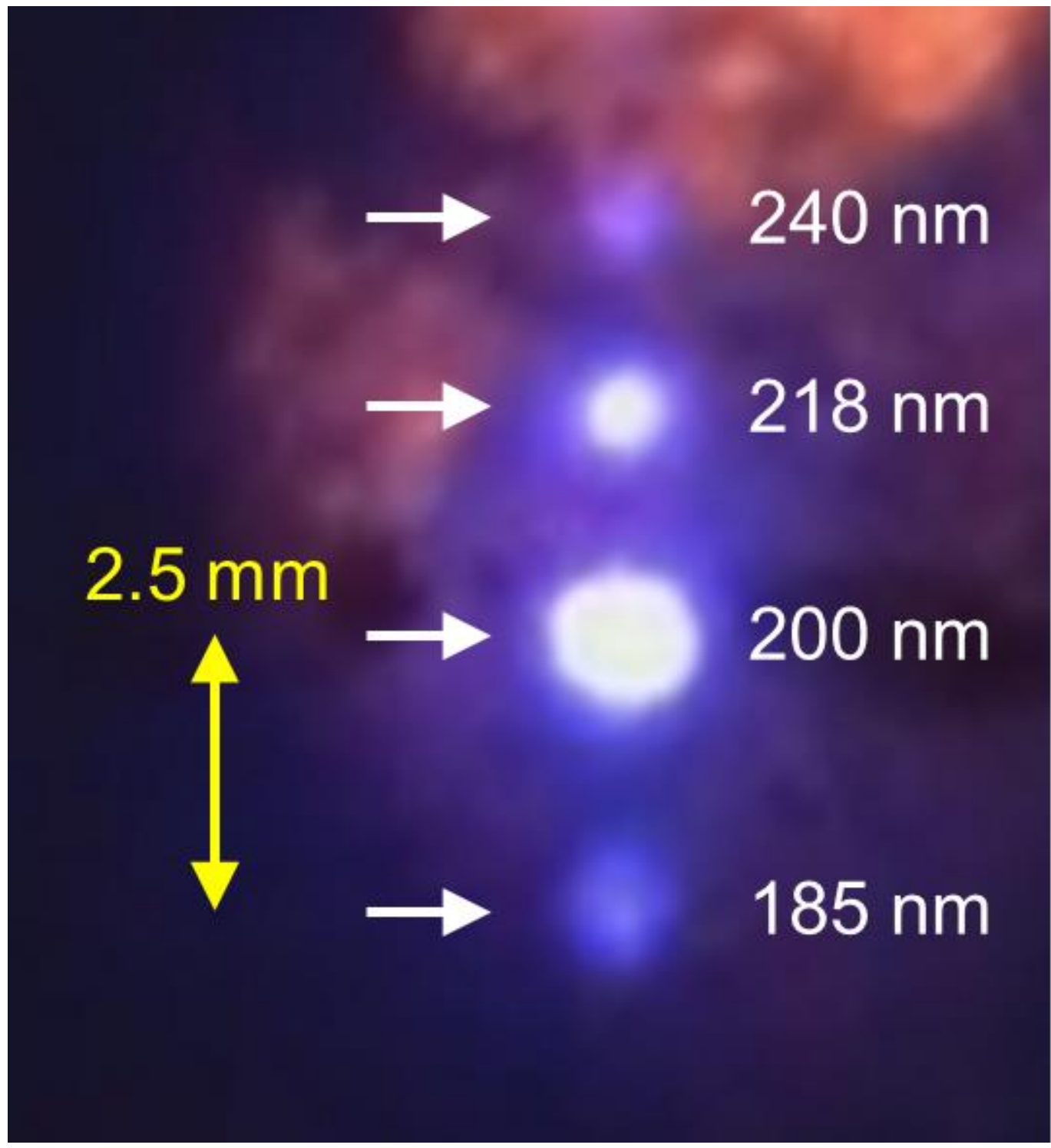


Figure S4. Absorption spectra calculated for pentachlorobenzene and the APAHs measured in this study. The chemical structure is shown as an insert in the figure. The wavelengths corresponding to the first excited energies $(E E)$, the ionization energy $(I E)$, and the half value of the ionization energy (IE/2) are indicated in the figure.
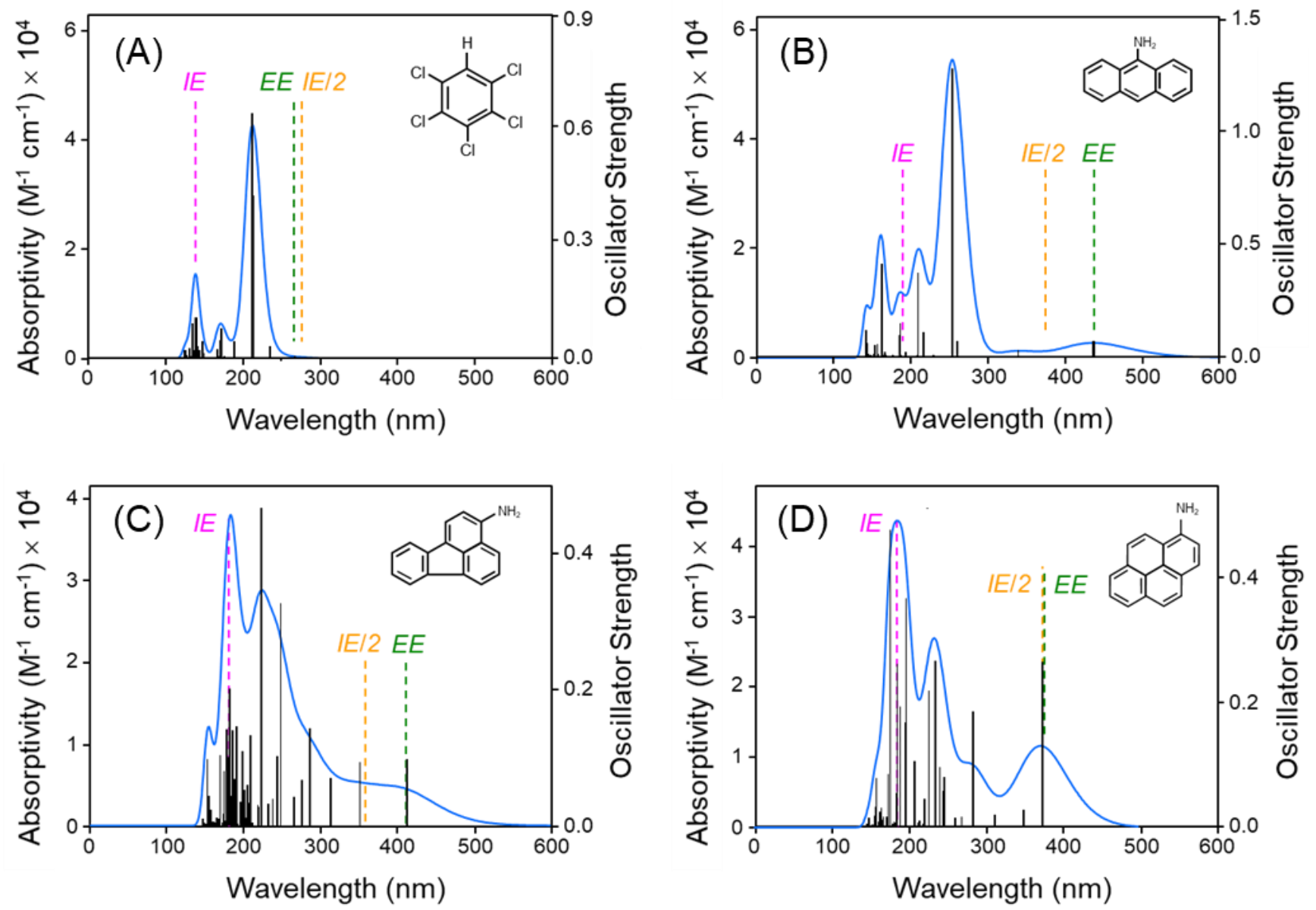